\title{
Antimicrobial Dialkylresorcinols from Pseudomonas sp. Ki19
}

\author{
Anton Pohanka, ${ }^{\dagger}$ Jolanta Levenfors ${ }^{\ddagger}$ and Anders Broberg* $*^{\dagger}$ \\ Department of Chemistry, Swedish University of Agricultural Sciences, P.O. Box 7015, SE-750 07 \\ Uppsala, Sweden; MASE Laboratories AB, P.O. Box 148, SE-751 04 Uppsala, Sweden \\ Anders.Broberg@kemi.slu.se. \\ * To whom correspondence should be addressed. Tel: +46-18-672217. Fax: +46-18-673392. \\ ${ }^{\dagger}$ Department of Chemistry, Swedish University of Agricultural Sciences \\ ${ }^{\ddagger}$ MASE Laboratories
}

Figure 1. HPLC chromatogram of lipophilic 95\% SPE eluate

Table 1. NMR Spectroscopic Data for $\mathbf{1}$ and $\mathbf{2}$

Compound Data for 3 (DB-2073) and 4 (resorstatin)

S3

Figure 2. ${ }^{1} \mathrm{H}$ NMR spectra of 1, 2, 3, 4 and 4a

S4 
Figure 1. HPLC chromatogram of lipophilic 95\% SPE eluate

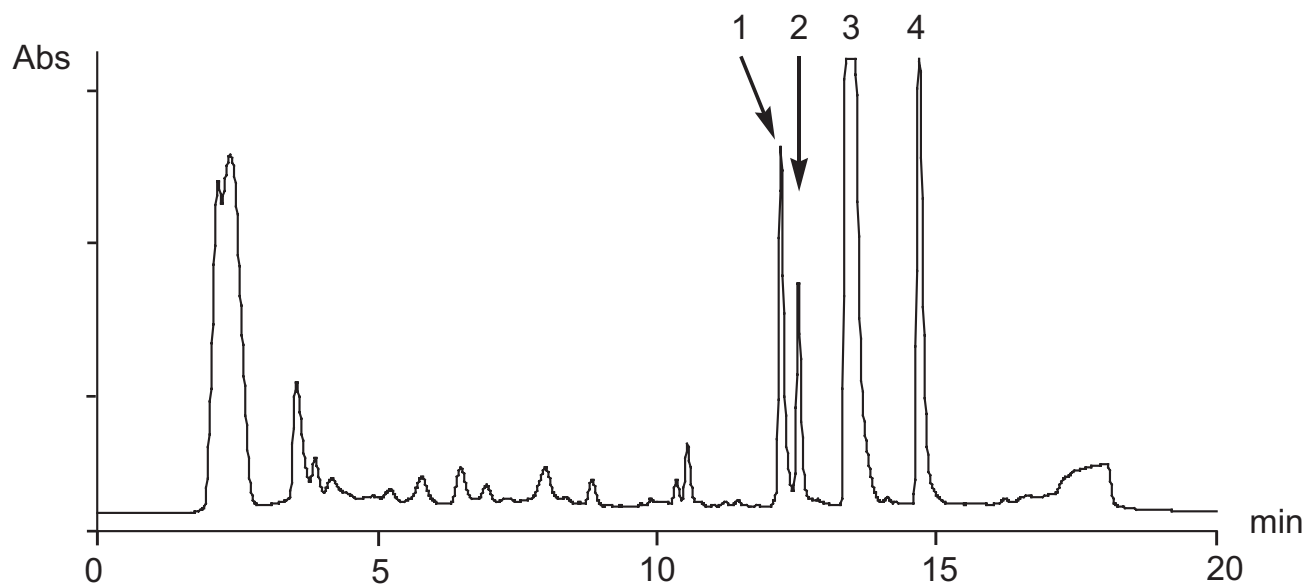

Numbers indicate peaks corresponding to compounds 1-4. HPLC system: 20 to $100 \% \mathrm{CH}_{3} \mathrm{CN}$ in $\mathrm{H}_{2} \mathrm{O}$ in $10 \mathrm{~min}$ with an $8 \mathrm{~min}$ hold at $100 \%$. The eluate was monitored at $210 \mathrm{~nm}$.

Table 1. NMR Spectroscopic Data $\left(\mathrm{CD}_{3} \mathrm{OD}, 100 / 400 \mathrm{MHz}\right)$ for 2,5-dialkylresorcinols 1 and 2

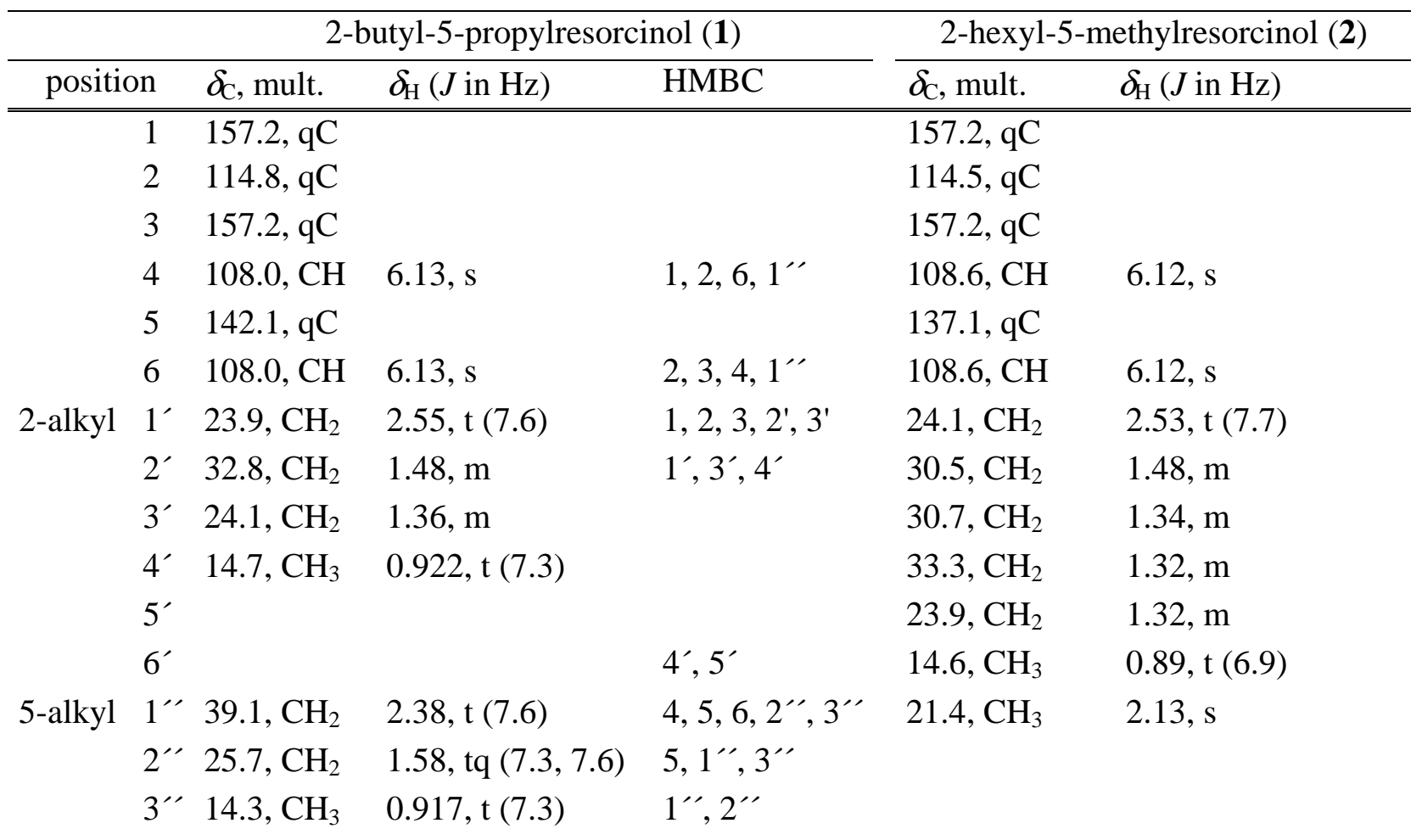


Compound Data for 3 (DB-2073) and 4 (resorstatin)

2-Hexyl-5-propylresorcinol [3 (DB-2073)]: white powder; UV $\left(\mathrm{CH}_{3} \mathrm{OH} / \mathrm{H}_{2} \mathrm{O}\right.$ 1:1) $\lambda_{\max }(\log \varepsilon) 207$ (2.67), 271 (2.28), 279 (sh) (2.20) nm; ${ }^{1} \mathrm{H}$ NMR $\left(\mathrm{CH}_{3} \mathrm{OH}-d_{4}, 400 \mathrm{MHz}\right) \delta 6.13$ (2H, s, H-4, H-6), 2.54 $\left(2 \mathrm{H}, \mathrm{t}, J=7.6 \mathrm{~Hz}, \mathrm{H}-1^{\prime}\right), 2.38\left(2 \mathrm{H}, \mathrm{t}, J=7.6 \mathrm{~Hz}, \mathrm{H}-1^{\prime \prime}\right), 1.58(2 \mathrm{H}, \mathrm{tq}, J=7.4,7.7 \mathrm{~Hz}), \mathrm{H}-2^{\prime \prime}, 1.49$ ( $2 \mathrm{H}$,

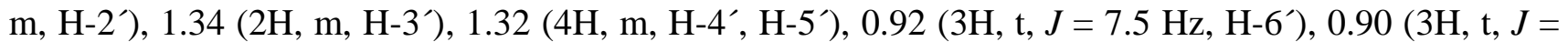
$\left.6.9 \mathrm{~Hz}, \mathrm{H}-3^{\prime \prime}\right) ;{ }^{13} \mathrm{C}$ NMR $\left(\mathrm{CH}_{3} \mathrm{OH}-d_{4}, 100 \mathrm{MHz}\right) \delta 157.1$ (C, C-1, C-3), 142.1 (C, C-5), 114.8 (C, C-2), 108.0 (C, C-4, C-6), $39.1\left(\mathrm{CH}_{2}, \mathrm{C}-1^{\prime \prime}\right), 33.2\left(\mathrm{CH}_{2}, \mathrm{C}-4^{\prime}\right), 30.7\left(\mathrm{CH}_{2}, \mathrm{C}-3^{\prime}\right), 30.5\left(\mathrm{CH}_{2}, \mathrm{C}-2^{\prime}\right), 25.7\left(\mathrm{CH}_{2}\right.$, C-2”), $24.1\left(\mathrm{CH}_{2}, \mathrm{C}-1^{\prime}\right), 23.9\left(\mathrm{CH}_{2}, \mathrm{C}-5^{\prime}\right), 14.6\left(\mathrm{CH}_{3}, \mathrm{C}-6^{\prime}\right), 14.3\left(\mathrm{CH}_{3}, \mathrm{C}-3^{\prime \prime}\right)$; diagnostic H2BC

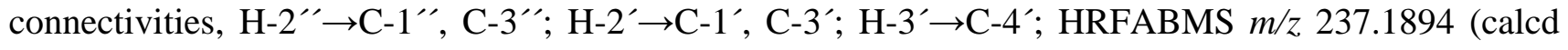
for $\left.\mathrm{C}_{15} \mathrm{H}_{25} \mathrm{O}_{2} 237.1855\right)$.

2-Hexyl-5-pentylresorcinol [4 (Resorstatin)]: white powder; $\mathrm{UV}\left(\mathrm{CH}_{3} \mathrm{OH} / \mathrm{H}_{2} \mathrm{O}\right.$ 1:1) $\lambda_{\max }(\log \varepsilon) 207$ (2.82), 271 (0.42), 280 (sh) (0.35) nm; ${ }^{1} \mathrm{H}$ NMR $\left(\mathrm{CH}_{3} \mathrm{OH}-d_{4}, 400 \mathrm{MHz}\right) \delta 6.14$ (2H, s, H-4, H-6), 2.54 $\left(2 \mathrm{H}, \mathrm{t}, J=7.6 \mathrm{~Hz}, \mathrm{H}-1^{\prime}\right), 2.40\left(2 \mathrm{H}, \mathrm{t}, J=7.6 \mathrm{~Hz}, \mathrm{H}-1^{\prime \prime}\right), 1.56\left(2 \mathrm{H}, \mathrm{m}, \mathrm{H}-2^{\prime \prime}\right), 1.49$ (2H, m, H-2'), 1.34

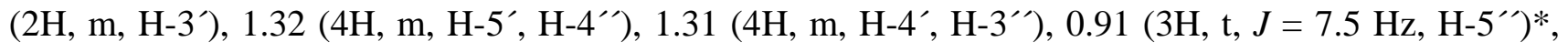
$0.90\left(3 \mathrm{H}, \mathrm{t}, J=6.9 \mathrm{~Hz}, \mathrm{H}-6^{\prime}\right) * ;{ }^{13} \mathrm{C} \mathrm{NMR}\left(\mathrm{CH}_{3} \mathrm{OH}-d_{4}, 100 \mathrm{MHz}\right) \delta 157.2(\mathrm{C}, \mathrm{C}-1, \mathrm{C}-3), 142.4(\mathrm{C}, \mathrm{C}-5)$, 114.8 (C, C-2), 108.0 (C, C-4, C-6), $36.9\left(\mathrm{CH}_{2}, \mathrm{C}-1^{\prime \prime}\right), 33.2\left(\mathrm{CH}_{2}, \mathrm{C}-3^{\prime \prime}\right), 32.8\left(\mathrm{CH}_{2}, \mathrm{C}-4^{\prime}\right), 32.3\left(\mathrm{CH}_{2}\right.$, C-2”'), $30.7\left(\mathrm{CH}_{2}, \mathrm{C}-3^{\prime}\right), 30.5\left(\mathrm{CH}_{2}, \mathrm{C}-2^{\prime}\right), 24.1\left(\mathrm{CH}_{2}, \mathrm{C}-1^{\prime}\right), 23.7\left(\mathrm{CH}_{2}, \mathrm{C}-5^{\prime}\right), 23.9\left(\mathrm{CH}_{2}, \mathrm{C}^{\prime} 4^{\prime \prime}\right), 14.6$ $\left(\mathrm{CH}_{3}, \mathrm{C}-5^{\prime \prime}\right)^{* *}, 14.5\left(\mathrm{CH}_{3}, \mathrm{C}^{\prime} 6^{\prime}\right)^{* *}$; diagnostic HMBC connectivities, H-2' $\rightarrow \mathrm{C}-3^{\prime} ; \mathrm{H}-3^{\prime} \rightarrow \mathrm{C}-4^{\prime}$; H2 " $\rightarrow$ C-3"'; C-4"; HRFABMS m/z 265.2146 (calcd for $\mathrm{C}_{17} \mathrm{H}_{29} \mathrm{O}_{2}$ 265.2168); **** may be interchanged. 
Figure 2. ${ }^{1} \mathrm{H}$ NMR spectra of $\mathbf{1}, \mathbf{2}, \mathbf{3}, \mathbf{4}$ and $\mathbf{4 a}\left(\mathrm{CD}_{3} \mathrm{OD}, 400 \mathrm{MHz}\right)$

1<smiles>CCCCc1c(O)cc(CCC)cc1O</smiles>

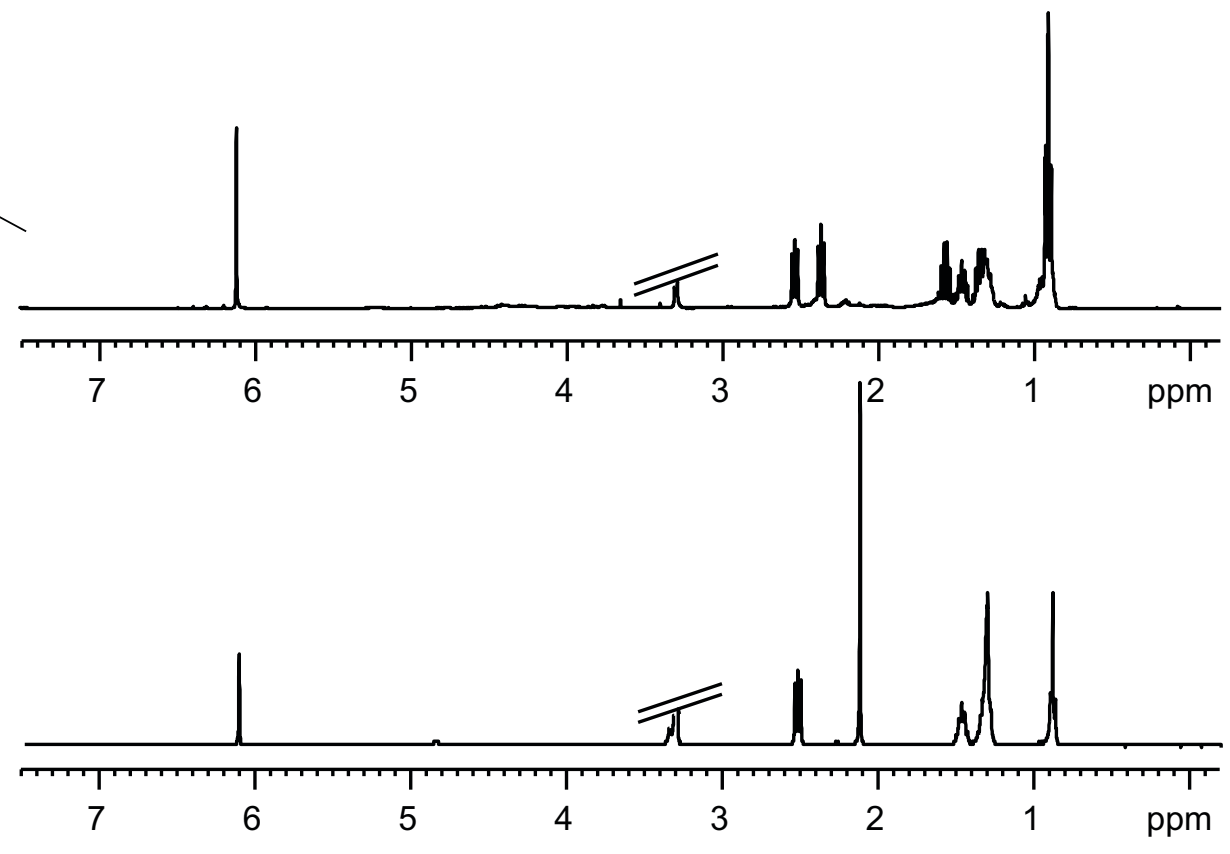<smiles>CCCCCCc1c(O)cc(C)cc1O</smiles>

3<smiles>CCCCCCc1c(O)cc(CCC)cc1O</smiles>

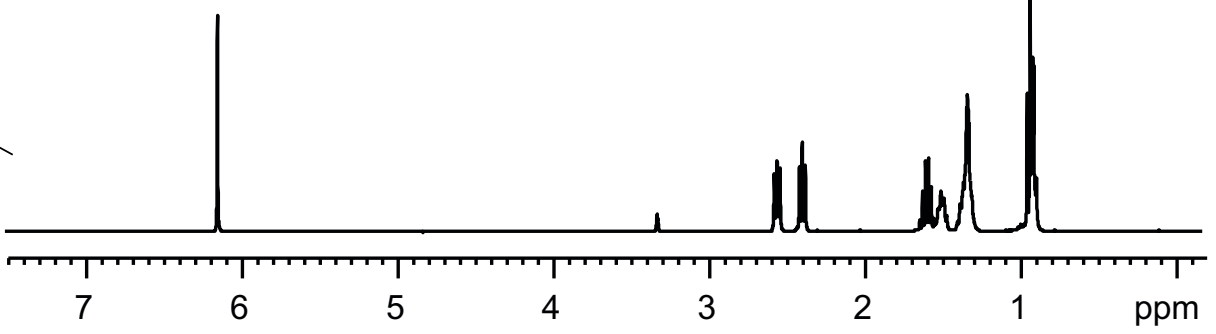

4

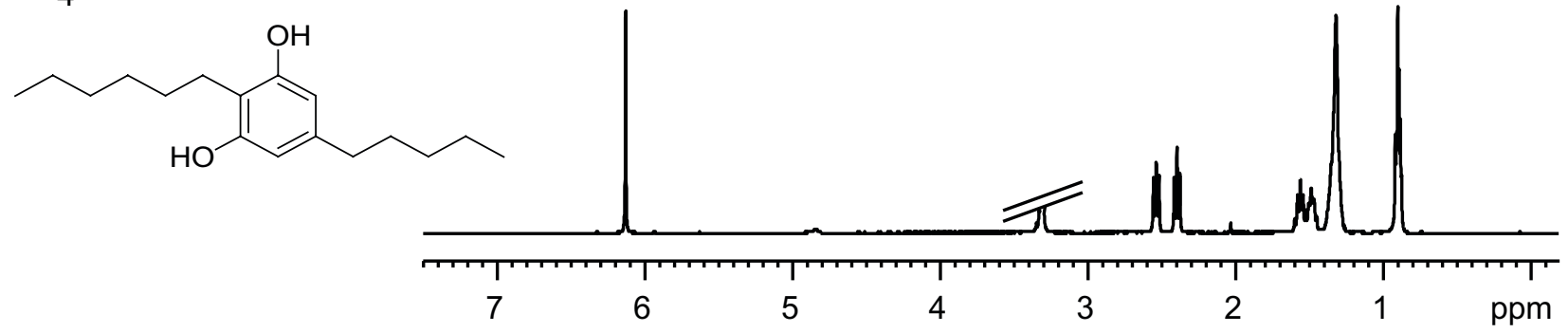

$4 a$

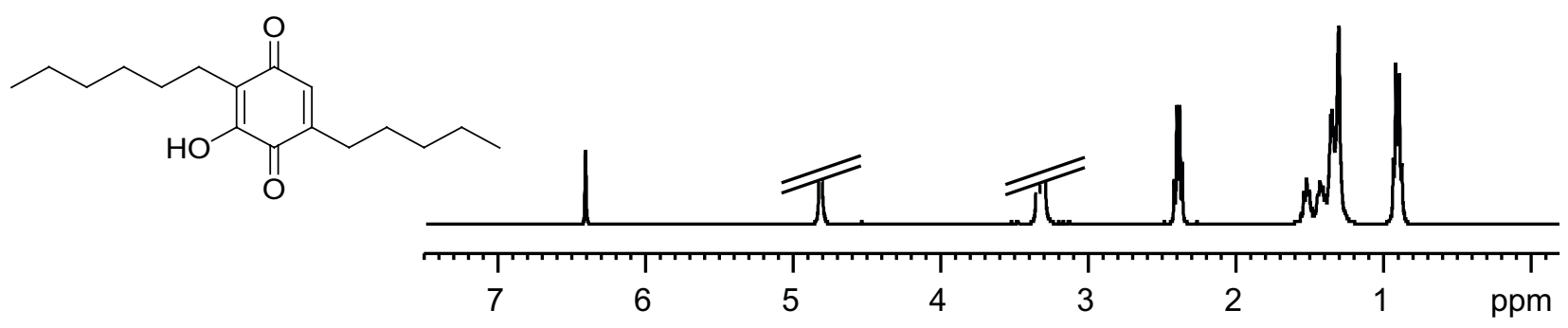

SH 